\title{
Aprendizaje con servicio voluntario en la formación inicial docente*
}

\author{
Volunteer service learning in initial teacher training \\ Aprendizado de serviço voluntário na formação inicial de professores
}

\author{
Williamson, Guillermo ${ }^{a}$ :Espinoza, Francisco ${ }^{b}$; \\ Ferreira, Solange ${ }^{c}$; Abello, Eduardo ${ }^{d}$
}

aDepartamento de Educación, Universidad de La Frontera, Temuco, Chile. Teléfono: 56-45-325366.

Correo electrónico: guillermo.williamson@ufrontera.cl

\author{
bUniversidad de La Frontera, Temuco, Chile. Correo electrónico: f.espinoza01@ufromail.cl \\ cUniversidad de La Frontera, Temuco, Chile. Correo electrónico: s.ferreira@ufromail.cl \\ dUniversidad de La Frontera, Temuco, Chile. Correo electrónico: edoabello@gmail.com
}

\begin{abstract}
RESUMEN
El artículo aborda el aprendizaje con servicio de estudiantes de pedagogía en comunidades educativas, entendiéndolo como alternativa y/o complemento que apoya a la formación inicial docente. Se fundamenta en la sistematización y una breve investigación exploratoria sobre el trabajo realizado por un grupo de estudiantes de pedagogía de la Universidad de La Frontera en dos comunidades educativas de la Región de la Araucanía: El "Hogar Wechekeche Inatu Kimun", ubicado en Temuco, y el "Complejo Educacional Maquehue", de Padre Las Casas. Se plantea, así, una alternativa a la formación inicial docente desarrollando proyectos en contextos reales.

Palabras clave: aprendizaje con servicio, formación inicial docente, voluntariado, educación liberadora.
\end{abstract}

\begin{abstract}
The article refers to service-learning of teaching program students in different educational communities, understanding this practical approach as an alternative and/or complement that supports their Initial Teacher Education. It is based on the systematization and brief exploratory research on the work performed by a group of teaching program students from Universidad de La Frontera in two different educational communities: "Hogar Wechekeche Inatu Kimun", located in Temuco, and, "Complejo Educacional Maquehue", located in Padre Las Casas. Thus, it develops an alternative methodology to the initial training for future teachers in real educational projects.
\end{abstract}

Key words: service-learning, initial teacher education, volunteerism, liberating education.

\section{RESUMO}

Aborda a aprendizagem em serviço de estudantes de Pedagogia em comunidades educativas, compreendendo-a como alternativa e/ou complementação da formação inicial docente. Fundamenta-se na sistematização e em uma breve pesquisa exploratória sobre o trabalho realizado por um grupo de estudantes de Pedagogia da Universidade de La Frontera, em duas comunidades educativas da Região de La Araucanía: o "Hogar Wechekeche Inatu Kimun", em Temuco, e o "Complejo Educacional Maquehue", de Padre Las Casas. Expõe uma alternativa para a formação inicial docente no desenvolvimento de projetos em contextos reais.

Palavras chave: aprendizagem em serviço, formação inicial docente, voluntariado, educação liberadora.

\footnotetext{
* Este artículo se basa en la ponencia presentada por los co-autores Abello, Espinoza y Ferreira en el Congreso de Profesores de Historia realizado en la Universidad de La Frontera en octubre de 2012, y en las evaluaciones de los proyectos realizadas por sus coordinadores junto con el académico responsable.
} 


\section{PRESENTACIÓN}

Este artículo referido al aprendizaje con servicios voluntarios, se fundamenta en la sistematización y una breve investigación exploratoria sobre el trabajo realizado por un grupo de estudiantes de pedagogía de Historia, Geografía y Educación Cívica en dos comunidades educativas de la Región de la Araucanía a partir de Convenios entre las instituciones educativas y la Universidad de La Frontera ${ }^{1}$. El Hogar Wechekeche Inatu Kimun, ubicado en Temuco y el Complejo Educacional Maquehue, de Padre Las Casas. Ambos tienen como característica principal la masiva presencia de estudiantes indígenas mapuche, su bajo nivel socioeconómico y escasas expectativas educacionales, todo ello plantea un gran desafío para los futuros docentes al enseñar los contenidos en contextos complejos. Estas experiencias se iniciaron el año 2011. El trabajo desarrollado por los estudiantes universitarios trata principalmente de apoyo pedagógico a través del reforzamiento de contenidos disciplinares y preparación para la Prueba de Selección Universitaria (PSU), además del desarrollo de actividades de acompañamiento personal a los estudiantes que participan en ambas instancias. El estilo de la narración es en muchos casos en primera persona plural, es decir, es un texto en que los participantes hablan desde su propia experiencia y significación de ella.

\section{INTRODUCCIÓN}

El trabajo que aquí se expone se organiza bajo el objetivo de presentar una propuesta de formación inicial docente basada en el aprendizaje con servicio en contextos reales. Esta experiencia por un lado busca el desarrollo de competencias pedagógicas, de gestión curricular y organizacional por parte de los futuros docentes, quienes se integran en las comunidades educativas para adquirir nuevas herramientas que le ayuden a formar una identidad profesional coherente con el rol transformador de los profesores en la sociedad actual. Por otro lado, se busca que los estudiantes adquieran competencias disciplinarias y transversales; así también, transformar las realidades de los estudiantes para formar en valores, fomentar la identidad territorial, desarrollar la idea de libertad, entendida como la capacidad de conocer críticamente los procesos que han configurado su realidad; de modo que puedan utilizarla como base para el encuentro de la felicidad. La autogestión del conocimiento se hace fundamental tanto, para la formación docente como la de los estudiantes y de las comunidades educativas en su conjunto; puesto que fomenta los procesos de indagación e incentiva la formación continua, para generar comunidades de conocimiento y de praxis que colaboren a los diversos actores involucrados en el "servicio educacional" en la generación de cambios que los conduzcan a la libertad de pensamiento, de decisión y de compromiso pedagógico, social y político.

\footnotetext{
Se trata de convenios de la Facultad de Educación, Ciencias Sociales y Humanidades y su Departamento de Educación con el Complejo Educacional Maquehue y con la Secretaría Regional Ministerial de Educación de la Región de La Araucanía, del Ministerio de Educación. Son dirigidos por el Profesor Dr. Guillermo Williamson del Departamento de Educación.
} 


\section{MARCO TEÓRICO}

La formación inicial docente ha pasado por una serie modificaciones y ajustes para poder dar respuesta a contextos cambiantes en una sociedad en donde el capitalismo global se ha instaurado modificando las relaciones sociales, culturales y económicas. En este sentido, la forma de entender los procesos educativos tanto de los futuros profesores y profesoras, como de los educandos debe cuestionarse constantemente ¿Para qué se forman los futuros docentes? Para esta investigación, la educación en el contexto sistémico actual será entendida como un método efectivo para mantener ciertas estructuras de poder asimétricas (clases dominantes-excluidas), que no permiten el desarrollo integral e igualitario (en cuanto a posibilidades) de las personas, generándose así estructuras de dependencia y sumisión:

este autoritarismo lo manifiesta la sociedad desde la educación para crear personas dependientes, autoritarias y competitivas que asumen las injusticias del sistema como algo natural a la humanidad, integrándonos en un sistema insolidario y que mantiene desigualdades, violencia, enfrentamientos y explotación (Cuevas, 2001: 3).

Considerando lo anterior, y conociendo la realidad chilena, en donde las evaluaciones estandarizadas han calado en todos los niveles el sistema educativo articulándose como mecanismos castigadores y que no miden la alteridad de los individuos y mucho menos características intrínsecas de cada sujeto, se hace necesaria entonces, la articulación de metodologías innovadoras y efectivas que sean conscientes de la realidad social que rodea a los actores sociales insertos en el sistema educativo, de manera tal de devolver la libertad a los educandos y de humanizar y dignificar la labor docente. La metodología propuesta, busca incorporarse dentro de este marco para poder integrar la teoría y la praxis en la formación de futuros pedagogos, dotándolos de una capacidad comprensiva con respecto a su rol humanizador y formador y potenciar la capacidad pro-social, es decir, la capacidad empática para poder realizar cambios reales en las comunidades donde se insertan. En este sentido, el aprendizaje con servicio, será entendido como:

una forma de educación basada en la experiencia, en la que el aprendizaje se produce a través de un ciclo de acción y reflexión gracias al cual los estudiantes trabajan con otros compañeros en un proceso de aplicación de lo que han aprendido a los problemas de la comunidad y, al mismo tiempo, reflexionan sobre la experiencia de perseguir objetivos reales para la comunidad e incrementar su propia comprensión y destrezas, es decir, desarrollan de manera conexa las múltiples dimensiones humanas y cultivan la responsabilidad cívica y social (Eyler y Gilers, 1999 Cit. en Francisco y Moliner, 2010: 71).

Así, el aprendizaje con servicio se transforma en una herramienta que funcionaría de forma "circular", en donde las relaciones entre profesores y estudiantes se vuelven más democráticas y horizontales, y se adquieren competencias curriculares (teniendo claro lo que se enseña es posible transmitirlo y hacerlo tangible) y cívicas, en donde el compromiso y la motivación personales para resolver los problemas reales en contextos reales, para el bien común de la comunidad, son los ejes centrales de la acción (Puig y Palos, 2006). El componente gravitante en esta propuesta, es el trabajo de los estudiantes universitarios en contextos reales, que salgan del control y la idealización de la cátedra 
académica, brindando una oportunidad de que los estudiantes puedan posicionarse como sujetos de cambio social aportando conocimientos y experiencias y enriqueciendo su saber académico volviéndose más competente, constructivo y proactivo durante su ejercicio laboral (Toledo y Reyes, 2010). Así, podemos definir como los principales componentes del aprendizaje con servicio, la generación de competencias de gestión, autonomía y didáctica, así como también el formar ciudadanos participativos y solidarios. Se diferencia de las actividades asistencialistas voluntarias, fundamentalmente porque los proyectos deben ser planteados de manera tal que los estudiantes participantes apliquen también sus conocimientos académicos adquiridos durante su formación académica (Tapia, 2001). Puig, Gijón, Martín y Rubio, señalan que "por un lado, el desarrollo de una acción de servicio transforma y da sentido a los aprendizajes y, por el otro, el desarrollo de un aprendizaje activo y significativo mejora la acción de solidaridad" (2011: 52). Lo que además permite la formación de competencias reflexivas y críticas, desarrollan el compromiso solidario y facilitan el ejercicio responsable de la ciudadanía. Pero por sobre todo, con un fuerte contenido reflexivo sobre la propia actividad e incidencia en el medio, es decir, tomar conciencia de que cada actividad realizada durante el proceso incide en las comunidades, por lo cual el nivel de responsabilidad adquiridos deben condecirse con la generación de actividades motivadoras y significativas para ambas partes, comunidad y estudiantes (Escamez, 1999; García Roca, 1994). De esta forma, exige la implicación personal y desinteresada en un proyecto que busca el bien común y que se adhiere de forma activa a la causa del otro. Al reconocer la carencia del otro, no basta con indignarse ante la injusticia, sensibilizarse ante el sufrimiento ajeno o criticar los problemas que padece un sector de la humanidad (Puig et al., 2011), sino que es necesario, según Freire (2011), actuar para poder alcanzar la libertad de forma real, perdiendo el miedo a vivirla.

Otro punto importante sobre la realización de un proyecto de aprendizaje con servicio, es la democratización del mismo, es decir, son proyectos en donde las temáticas a trabajar son escogidas por los estudiantes, luego de un diagnóstico previo de las necesidades de la comunidad a intervenir. Como segundo punto, son proyectos realizados con un alto grado de autonomía, en donde los profesores no intervienen supervisando y calificando el proceso, sino que las evaluaciones nacen de un proceso de reflexión conjunta entre los actores, por lo que hace necesario la generación de un clima de mayor confianza con el estudiantado que permita un cambio en las relaciones entre el profesorado y el alumnado al generarse las condiciones necesarias para un diálogo más igualitario y sincero (Francisco y Moliner, 2010).

Sin embargo, la lógica que guía los procesos de formación inicial docente se basa en una visión técnica y prescriptiva de la naturaleza del conocimiento. La visión del profesor como un técnico favorece la fragmentación del conocimiento teórico prescriptivo y absoluto, luego de lo cual, se pretende que se "vacíen" esos conocimientos durante la práctica profesional; no se reconoce la multidimensionalidad de los fenómenos educativos ni la posibilidad de que los profesores sean productores de conocimiento al reflexionar sistemáticamente durante su quehacer docente y sobre este (Labra, 2011). De modo que la capacidad innovadora y creativa se ve castrada al desconectar o parcelar el conocimiento teórico del práctico y cuando se articulan, se realiza de forma paralela, convirtiendo el ejercicio docente en una práctica carente de sentido, sin considerar que la formación inicial docente constituye uno de los elementos básicos en el desarrollo profesional de los profesores y profesoras constituyendo uno de los principales instrumento para alcanzar 
una mejor calidad de la enseñanza, pues las competencias y los recursos que se adquieren en dicho proceso son los que van a servir de base para la formación permanente del profesorado (Palomera, Fernández-Berrocal y Brackett, 2008).

Por otro lado, la educación universitaria en los últimos años se ha centrado en el fortalecimiento del rol de las universidades dentro de los territorios que las contienen, en este sentido, la UNESCO (1998: en línea) señala que:

la Educación Superior debe reforzar sus funciones de servicio a la sociedad, y más concretamente sus actividades encaminadas a erradicar la pobreza, la intolerancia, la violencia, el analfabetismo, el hambre, el deterioro del medio ambiente y las enfermedades, principalmente mediante un planteamiento interdisciplinario y transdisciplinario para analizar los problemas y las cuestiones planteadas.

Y además el enfoque basado en competencias se alza como una respuesta a los lineamientos de la UNESCO, generándose el llamado "Aprendizaje Basado en Competencias, las cuales son definidas por la OCDE como la combinación de habilidades prácticas, conocimientos, motivación, valores éticos, actitudes, emociones y otros componentes sociales que se movilizan conjuntamente para lograr una acción eficaz" (Hersh et. al., 1999 Cit. en Francisco y Molliner, 2010: 70).

En este marco, el aprendizaje con servicio parece ser una respuesta eficaz a lo que se pretende sea la educación superior, volviéndose una alternativa válida para concretar la conexión universidad-territorio y la adquisición de competencias si se enmarca dentro de la formación inicial docente como una experiencia (proyecto) de practica temprana. Diversas investigaciones, han buscado sintetizar y sistematizar las competencias obtenidas al realizar proyectos de aprendizaje con servicio, en esta línea, Furco (Cit. en Puig et al., 2011: 62) señala:

Tabla 1. Impacto educativo del aprendizaje con servicio

\begin{tabular}{|c|c|}
\hline \multicolumn{2}{|r|}{ Impacto educativo del aprendizaje servicio } \\
\hline $\begin{array}{l}\text { Académico y } \\
\text { cognitivo }\end{array}$ & $\begin{array}{l}\text {-Aumento del rendimiento en pruebas estandarizadas. } \\
\text {-Mayor desarrollo de conocimientos conceptuales y competencias. } \\
\text {-Mayor asistencia, motivación respecto a la escuela y retención. } \\
\text {-Mejores notas promedio. } \\
\text {-Mayor habilidad para analizar y sintetizar información compleja. }\end{array}$ \\
\hline $\begin{array}{l}\text { Formación } \\
\text { cívica }\end{array}$ & $\begin{array}{l}\text {-Mayor comprensión de la política y de las actividades gubernamentales. } \\
\text {-Mejor participación en la comunidad y en las cuestiones públicas. } \\
\text {-Mejor ejercicio de la ciudadanía y de la responsabilidad ciudadana. } \\
\text {-Mayor conciencia y comprensión de cuestiones sociales. } \\
\text {-Compromiso con el servicio comunitario. }\end{array}$ \\
\hline $\begin{array}{l}\text { Vocacional y } \\
\text { profesional }\end{array}$ & $\begin{array}{l}\text {-Ampliación de la conciencia y de las opciones vocacionales. } \\
\text {-Mejora de las competencias profesionales. } \\
\text {-Mayor comprensión de la ética del trabajo. } \\
\text {-Mejor preparación para el mundo del trabajo. }\end{array}$ \\
\hline
\end{tabular}




\begin{tabular}{|c|c|}
\hline \multicolumn{2}{|r|}{ Impacto educativo del aprendizaje servicio } \\
\hline Ético y moral & $\begin{array}{l}\text {-Mayor exposición a nuevos puntos de vista y perspectivas. } \\
\text {-Cambios positivos en el juicio ético. } \\
\text {-Mayor habilidad para tomar decisiones independientes respecto a cuestiones } \\
\text { morales. }\end{array}$ \\
\hline Personal & $\begin{array}{l}\text {-Ampliación de las cualidades y competencias para el liderazgo. } \\
\text {-Mayor autoestima. } \\
\text {-Mayor conocimiento de sí mismo. } \\
\text {-Mayor resiliencia. } \\
\text {-Mayor eficacia personal. }\end{array}$ \\
\hline Social & $\begin{array}{l}\text {-Mayor compañerismo entre estudiantes. } \\
\text {-Mayor habilidad para trabajar en equipos o para trabajar con otros. } \\
\text {-Capacidad para desechar los prejuicios. } \\
\text {-Mejora de las conductas prosociales. }\end{array}$ \\
\hline
\end{tabular}

Además, trabajos realizados en Venezuela (Toledo y Reyes, 2010) y España (Francisco y Moliner, 2010), utilizando técnicas cuantitativas y cualitativas respectivamente, han podido demostrar como los estudiantes han construido su propio aprendizaje ayudando a sus comunidades desde la autogestión del conocimiento, la generación de redes de apoyo y la creación de trabajo cooperativo en comunidades de aprendizaje para poder resolver problemas sociales, educando para la libertad, con libertad, en base a la autonomía de los sujetos para auto formarse en el ser más liberador (Freire, 2011), a partir de la experiencia de aprender a ser uno mismo "nos referimos al trabajo formativo que cada individuo realiza sobre sí mismo para liberarse de ciertas limitaciones, para construir una manera de ser deseada y para lograr el mayor grado posible de autonomía y de responsabilidad" (Puig et al., 2011: 49).

Por otro lado, el aprendizaje con servicio, se alza como una forma distinta de entender las relaciones sociales y por ende, la escuela será entendida también de manera diferente, ya no más como una forma de reproducción de la desigualdad y de las estructuras de poder que niegan la naturaleza integral del ser humano, basada en la solidaridad y la generación de vínculos comunitarios de aprendizaje que también inciden políticamente ya que traspasa los aspectos netamente curriculares, para buscar inculcar valores e intentar cambiar las estructuras sociales. Para ser más explícitos "su horizonte pretende llegar un poco más lejos, contribuir a la formación de ciudadanos críticos y comprometidos, a la transformación de las situaciones de injusticia y a la construcción de sociedades democráticas y participativas" (Puig et al., 2011: 64).

El principio de lo anterior, se basa en preceptos antiautoritarios, cuyo "objetivo final del antiautoritarismo pedagógico es conseguir que los educandos 'sean dueños de su propia vida y que no se dejen oprimir ni explotar', poniendo en práctica el libre pensamiento y la autonomía moral" (García, 1986 Cit. en Cuevas, 2001: 4). Como se mencionó anteriormente, el aprendizaje con servicio, tiene sus bases en la autonomía de los estudiantes de pedagogía, pero también, es necesario, traspasar esa autonomía a los receptores de la intervención, de modo que sea posible la toma de conciencia de todos los actores involucrados en el proceso educativo. Se entenderá autonomía como "autonomía del individuo, en contra de las dependencias jerarquizadas y asumidas, cada individuo tiene derechos y obligaciones asumidas voluntariamente, responsabilidad colectiva y respeto. Las personas 
afrontan sus propios problemas, crean sus propias convicciones y razonamientos" (Colectivo Paideia) $)^{2}$. Por lo tanto, la evaluación castigadora será reemplazada por la reflexión colectiva y personal constante, evaluando los procesos sociales como avances hacia la consecución de la libertad para poder desarrollarse de los educando y educadores. Otros principios básicos del actuar dentro de los proyectos de aprendizaje con servicio, en términos de lo que plantea Cuevas (2001), son:

- Libertad del individuo. Libertad del individuo pero colectiva es decir teniendo en cuenta a los demás y desde la responsabilidad a vivir en grupo.

- En contra de la autoridad. Nadie manda a nadie todo se hace por compromisos asumidos y desde la decisión colectiva, abierta y sincera.

Es decir, cada uno de los integrantes de los proyectos, deben actuar acorde al respeto hacia sí mismos y a los demás integrantes del proyecto, lo que implica no abandonarlo, sin razones justificables, ya que se trata de compromisos adquiridos tanto con los pares, como con los actores involucrados de las comunidades educativas donde se instauren los proyectos.

En este sentido, la libertad se construye mediante la libertad; solo mediante una educación que enseña al educando a ser libre se pueden conseguir personas libres. Se entenderá en este sentido, la libertad como un proceso constante, que se alcanza luego de la toma de conciencia sobre la condición de opresión, en este sentido, se busca educar para la libertad, pero también para el compromiso (Cuevas, 2001) ya que no se trata de cambiar roles (opresor-oprimido/oprimido-opresor) sino de un proceso de liberación colectivo, comprometido con la liberación de la comunidad completa. Para esto, el maestro debe tener conciencia de que su papel es el de acompañar al estudiante, respetando su individualidad, y que el verdadero maestro no se caracteriza por sus títulos y diplomas, sino porque sigue educándose mediante la voluntad de perfeccionarse sin fin (Cuevas, 2001).

Un punto de partida para lo anterior, es intentar transformar la enajenación social de los estudiantes, producto de su inserción en un sistema que busca el adormecimiento de la alteridad, ese debe ser el primer paso hacia el largo camino de la liberación, en palabras de Araujo, “(...) esa cultura del ocio y diversión es el reverso de la moneda de la lucha inevitable y terrible de quienes buscan empleo. No es la solidaridad lo que caracteriza a esta juventud, sino una falta de sentido común en cuanto a los mercados consumidores de la diversión" (Araujo, 2004: 217). El aprendizaje con servicio, busca volver a comprometer valores de solidaridad con y hacia la comunidad, educando en libertad para la libertad, intentando de ser un referente de admiración, un modelo a seguir para los receptores del servicio, se alza en este sentido, como una alternativo al modelo tradicional y tecnocrático, una de tantas que buscan humanizar la educación.

\section{MARCO METODOLÓGICO}

La investigación presentada, se enmarcó dentro del paradigma cualitativo, ya que permitió conocer y sistematizar las percepciones y motivaciones de los individuos que han sido parte de la investigación de forma más profunda.

2 Recuperado el 24 de octubre de 2012 desde http://www.paideiaescuelalibre.org/Pedagogia\%20Libertaria.htm 
Los métodos empleados, principalmente fueron dos, el principal es el método etnográfico, basándose principalmente en la observación participante, la cual, de acuerdo a Amezcua (2000: 31), “es la base de la investigación etnográfica, que se ocupa del estudio de los diferentes componentes culturales de las personas en su medio: las relaciones con el grupo", de esta forma, según Kawulich (2005) la observación participante permite vislumbrar datos que no se podrían obtener por otros medios, logrando una mayor comprensión de lo que está ocurriendo y por ende una mejor comprensión del contexto y especialmente del fenómeno que se estudia. La observación participante se ha llevado a cabo durante 1 año y 6 meses aproximadamente. Por otro lado, se utilizaron entrevistas semi-estructuradas, cuya muestra para la entrevista fue de tipo intencional, ya que según Martínez (2006) ella permite elegir los casos más representativos y recurrir a las personas u organismos que entreguen la mayor cantidad de información útil.

Como señalamos, esta investigación sobre el aprendizaje con servicios, se basa en el trabajo realizado por un grupo de estudiantes de pedagogía en dos comunidades educativas de la región de la Araucanía (Hogar Wechekeche Inatu Kimün y Complejo Educacional Maquehue), con características como la masiva presencia de población mapuche, el bajo nivel socioeconómico de los estudiantes, las escasas expectativas educacionales de estos, entre otras, las cuales plantean un gran desafío para los futuros docentes al enseñar los contenidos en contextos complejos. El trabajo se basa principalmente en apoyo pedagógico a través del reforzamiento de contenidos disciplinares y preparación para PSU, además del desarrollo de actividades de acompañamiento a los estudiantes que participan en ambas instancias.

\section{DESARROLLO O RESULTADOS}

\subsection{OBSERVACIÓN ETNOGRÁFICA}

\subsubsection{Trabajo en Complejo Educacional Maquehue}

El Proyecto Maquehue consiste en un preuniversitario realizado por estudiantes de pregrado de la UFRO, donde, lo destacable es el contexto en el que se trabaja. Es un establecimiento de enseñanza media humanista-científica rural, con la mayoría de los 50 estudiantes de escasos recursos y de origen mapuche, con grandes falencias en su formación básica (incluso dificultades en la lectura y operaciones básicas) y pocas o nulas proyecciones para el futuro. El trabajo se consideraba todo un reto pues no es un ambiente para el que los estudiantes universitarios sean o estén preparados pues no reciben los conocimientos necesarios para organizar un proyecto de voluntariado, sin recursos desde el inicio. En base a la realidad el equipo se planteó ayudar en el manejo de contenidos y a la vez apoyar a los estudiantes a mejorar sus expectativas y la confianza en sus capacidades. En Liceo también ha habido estudiantes de pedagogía en sus actividades de prácticas profesionales integradas a los equipos de trabajo voluntario.

Primeros pasos: El trabajo se inició conociendo la realidad del establecimiento por medio de dos vías: reuniones con el director y asistencia a los Consejos de Profesores, con la finalidad de tener la visión de todo el equipo docente-estudiantil sobre el proyecto, lo que se esperaba y su visión sobre la realidad del liceo. En los profesores no había un 
planteamiento unificado sobre lo que se quería lograr, no se sabía si sería un reforzamiento, preuniversitario u otra actividad; al final se optó por un preuniversitario que además cumpliera la función de orientar e incentivar a los estudiantes, desde la mirada de personas más cercanas a su edad que entiendan su forma de entender la realidad.

Otra actividad paralela la constituyó el reunir al equipo de trabajo, donde por razones de tiempo y responsabilidad se decidió que debían ser dos personas por área, existiendo dificultades para reunir al equipo debido al poco contacto con estudiantes de distintas carreras, por lo que no se conocía a las personas a las que más les pudiera interesar el proyecto. Esta situación se fue subsanando con el tiempo, logrando reunir al equipo. Se puso énfasis en que el trabajo fuese realizado por estudiantes que tuviesen interés de cumplir un rol o actividad social, utilizando como primera medida de selección el miedo, ya que se planteaba el contexto en que se trabajaría con dificultades mucho mayores a las reales, lo que permitió filtrar a personas que no estaban dispuestas a hacer un sacrificio más allá de lo mínimo. Una condición anexa es que debería existir el compromiso de que este trabajo no debería afectar las calificaciones ni los tiempos dedicados a los estudios obligatorios de su formación.

Planificación y proyección: Reunido el equipo y decidido por parte del liceo cual sería la labor de los estudiantes, se inició la planificación, en primer lugar era necesario planificar los horarios de asistencia, los que por sí solos significaban una dificultad, ya que se debía hacer coincidir el horario del liceo con el de los estudiantes del proyecto, tomando en cuenta que el tiempo de viaje superaba la hora. Este aspecto significo por parte del equipo del proyecto organizarse en sus tiempos y en su disponibilidad horaria, algo que era una gran dificultad debido al difícil semestre que se iniciaba. Además esta organización consideraba un horario para reuniones del equipo, a fin de ir analizando las situaciones, problemas y retos que surgen en el camino, además de acercar el equipo de trabajo tratando de lograr un trabajo de apoyo entre las distintas disciplinas.

Por otra parte era necesario trabajar los contenidos a tratar, ordenarlos para saber qué era lo primordial, debido al poco tiempo con el que se contaba, esto fue realizado por los encargados de cada área, ya que es muy poco lo que puede opinar el equipo en general sobre un área que no maneja, esto en general fue un problema, ya que no poseíamos herramientas para poder discriminar en la importancia de contenidos en cada contexto, utilizándose como método de selección las áreas con mayor dificultad, lo que se obtuvo por medio de pruebas.

Resultados del primer año: Este primer año de trabajo a nivel de resultados cuantificables no fue muy provechoso, surgiendo en un primer momento un sentido de fracaso en parte del equipo, ya que tenían mayores expectativas sobre su desempeño y el resultado en los estudiantes, se tenían mayores expectativas, cuestionándose cada uno sus propias capacidades para trabajar, algo que como equipo debimos enfrentar reconociendo a fono nuestras debilidades y fortalezas.

Al realizar un balance como equipo del trabajo se lograron sacar a la luz las fortalezas, las debilidades y los logros de ese primer año:

Fortalezas: Lo que más ayudó al desarrollo del trabajo durante este primer año fue el gran interés y la importancia que le entregó el equipo en general al trabajo, además de 
la buena disposición y buenas relaciones tanto con los profesores como con la dirección del establecimiento, esto dio una buena base para iniciar el trabajo y continuarlo frente a las dificultades que surgían.

Debilidades: La primera debilidad que notamos fue la poca preparación que teníamos para trabajar en un proyecto como el que se desarrollaba y en general para enfrentarnos a la realidad educativa, el no saber planificar ni ordenar los tiempos, nula experiencia en trabajo interdisciplinario, no saber trabajar en el aula de clases y menos aun en contextos especiales. Es decir, la universidad nos entregó el conocimiento de las especialidades y algunas herramientas básicas, la que fueron insuficientes para realizar el trabajo.

Logros: El mayor logro a los ojos del equipo de trabajo fue haber iniciado y mantenido el proyecto pese a las dificultades que se presentaban (semestre comprimido, poco tiempo, falta de experiencia, etc.), se tuvo la capacidad de organizar el trabajo y llevarlo a cabo, se obtuvo experiencia de organizar un proyecto y las dificultades que esto implica, además se fue adquiriendo la capacidad de dar respuesta a los problemas inmediatos sin que esto afectara el funcionamiento general.

Segundo año: El inicio del trabajo del primer año fue más rápido, en primer lugar, ya se tenía la experiencia del año anterior por lo que se sabía qué hacer y por otra parte las circunstancias de inicio del trabajo fueron mejores. Además reunir el equipo fue más fácil, ya que existía una mayor red de contactos para poder llenar los cupos de los que se retiraban, dejando esta labor y haciendo responsable a cada equipo de dejar sus remplazos.

Se logró avanzar en una planificación mas estructurada en cada área, planteándose los tiempos necesarios para cada materia y las dificultades de la enseñanza, principalmente marcadas por los magros conocimientos previos de los estudiantes, su poca dedicación y el contexto de vulnerabilidad, tres situaciones para las que no preparan dentro de la universidad.

El trabajo se ha ido realizando con normalidad y ya se han notado cambios en los estudiantes participantes, principalmente en la habilidad que van adquiriendo para manejar las diferentes situaciones, tomando además confianza en si mismos y conociendo sus debilidades. Las reuniones que se han llevado a cabo se han vuelto más profundas, son menos los temas coyunturales que se tratan y ya se empieza a pensar en situaciones más complejas, como la orientación a los estudiantes y sus expectativas, para las cuales tenemos muy poca preparación, se busca además avanzar en el trabajo a largo plazo, ya que el proyecto se consolidó, por lo que es necesario darle continuidad en el tiempo con nuevos coordinadores y estudiantes de pregrado que planteen los planes para el próximo año, ya que el equipo con el cual partió el proyecto se retira debido a que se encuentran terminando sus carreras, debiendo dejar sus remplazos a los que deben apoyar con su experiencia, para poder avanzar así en un trabajo con buenas bases.

\subsubsection{Trabajo en el proyecto de colaboración con Hogar Indígena "Wechekeche Inatu Kimun"}

El proyecto de Apoyo Pedagógico en el Hogar de Estudiantes Indígenas de Enseñanza Media "Wechekeche Inatu Kimun" se da inicio tras el acuerdo del Proyecto de Colaboración 
entre el Proyecto Kelluwün del Departamento de Educación ${ }^{3}$ y la Secretaría Regional Ministerial de Educación de la Araucanía. El proyecto está enfocado en tres áreas de apoyo a los estudiantes, que son: el área de refuerzo de competencias académicas; el área de desarrollo de la identidad cultural e intercultural; y el área de desarrollo personal. Uno de los principales objetivos que se busca con este proyecto, es la formación en valores y de hábitos de estudio en los estudiantes, que valoren su formación escolar para que en un futuro próximo retornen a sus comunidades como profesionales y colaboren con el desarrollo de la misma y su entorno más cercano.

En este proyecto se atiende a estudiantes de enseñanza media que conviven en el hogar indígena tras su jornada escolar. Son estudiantes que asisten regularmente a establecimientos educacionales en Temuco y Padre las Casas, como el Liceo Pablo Neruda, Liceo Industrial de Temuco, Liceo Particular Comercial Temuco, Liceo Técnico Temuco, Liceo Comercial del Desarrollo, entre otros. Esta variedad en estudiantes de distintos establecimientos educacionales plantea un desafío mayor al atender una alta diversidad, que hace necesaria una buena preparación y coordinación de las clases.

Dicho Proyecto de Colaboración requiere la conformación de un equipo de trabajo de estudiantes de pedagogías, de los últimos años de formación, que coordinara y planificara las actividades de apoyo pedagógico que se realizarían en el Hogar Indígena.

Entre las dificultades que se tuvo al empezar los trabajos, una fue sortear la falta de espacios apropiados para la realización de las clases, pero con el tiempo se fueron habilitando algunas salas que facilitaron la concentración, la participación y el desarrollo de las clases; también lo fue el constituir los horarios de las clases, ya que al ser de distintos establecimientos educacionales distintos, diferían los horarios de los estudiantes, por lo que esa diversidad se tuvo que coordinar con la disponibilidad de tiempo del equipo de trabajo. Formando así grupos de trabajo divididos por curso y por disponibilidad horaria, a los cuales se les asignaba un docente de cada disciplina.

En sus inicios, comenzando el segundo semestre de 2011, durante las movilizaciones estudiantiles de ese año, el proyecto contó con un equipo de 9 estudiantes de pedagogía, tres de historia, tres de matemáticas, uno de lenguaje y dos de ciencias. El trabajo con los estudiantes del hogar indígena comienza a mediados de septiembre del 2011, agrupando en los cursos a los niños que voluntariamente quisieran participar de las clases, por lo que de los más de 70 estudiantes del Hogar, nuestro equipo trabajaba con alrededor de 30 estudiantes distribuidos en los distintos niveles de enseñanza.

Las expectativas que se tenían con respecto a los resultados del proyecto en los estudiantes ese año, eran bajas, ya que era poco lo que se podía hacer en tres meses antes que terminara el año escolar. Pero la idea era sentar bases para el trabajo que se realizaría en el 2012 durante todo el año, además de conocer el funcionamiento del Hogar y de motivar a que más jóvenes participen de las clases.

La evaluación de esos meses de trabajo arrojó grandes desafíos que no mermaron el compromiso del equipo de trabajo. Entre esos desafíos, estaba el incorporar más estudiantes de pedagogía al equipo de trabajo, mejorar los aspectos que estaban más débiles como la didáctica, el manejo de grupo, etc.

Proyecto de Extensión de la Universidad de La Frontera: "Programa Kelluwün: Educación, comunicaciones, interculturalidad y desarrollo" ( $\left.{ }^{\circ} 016 / 2012\right)$. 
El proyecto se retoma a inicios de abril de 2012 con un equipo de trabajo mayor, actualmente participan activamente del proyecto dieciocho estudiantes de pedagogía, atendiendo a las necesidades que presenten los estudiantes del hogar en las distintas disciplinas: 4 de matemáticas; 6 de ciencias; 3 de Historia; 5 de lenguaje.

Esta vez, en coordinación con la dirección del Hogar, se buscó que la participación en los refuerzos de contenidos fuera obligatoria, pero los incentivos no fueron suficientes para motivar la participación de los estudiantes. Por lo que se llegó al consenso de que la participación sería voluntaria.

Así, en las reuniones mensuales del equipo de trabajo, se ha ido evaluando y coordinando las actividades según las necesidades que surgen con el desarrollo de las clases en cada disciplina, dejando a criterio de cada futuro docente los pasos a seguir con sus respectivos cursos. La observación etnográfica permite dar cuenta de un proceso muy particular, en el que los estudiantes de las pedagogías que participan haciendo clases de reforzamiento valoran la experiencia como una oportunidad de formación complementaria y donde van evidenciando sus fortalezas y debilidades como futuros docentes. En donde el compromiso -en algunos más, en otros menos- es la base del trabajo con los estudiantes de enseñanza media que conviven en el hogar.

Entre los logros que ha sido posible dar cuenta tras más de un año de trabajo, está la importancia que los estudiantes del hogar le dan al trabajo de los futuros docentes, valorando la dedicación y el compromiso con la pedagogía, ya que la voluntad y el propio compromiso es el principal motor de estos proyectos. Se destaca este logro porque se ha tenido que lidiar con la constante desmotivación que algunos educandos tienen por estudiar o aprender, desmotivación que muchas veces incide en el desempeño académico de cada uno de ellos y afectando más de alguna vez incluso al equipo de trabajo.

En cuanto a las debilidades que se perciben a raíz del trabajo en este proyecto educativo, está la poca preparación para atender aspectos relacionados con la formación personal de los educandos. Puesto que el trabajo se realiza en un contexto donde, además de la desmotivación que se mencionaba anteriormente, incide también las escasas expectativas educacionales que tienen los jóvenes del hogar. Lo que hace necesario un acompañamiento más presente y particularizado, pero que muchas veces escapa de las facultades que como docentes en formación se tiene.

\subsection{ANÁLISIS DE ENTREVISTAS}

Las entrevistas fueron realizadas con la finalidad de corroborar los resultados de la observación del desarrollo de los proyectos y las diferencias y cambios que iban sucediendo en los participantes de los proyectos. Se aplicó también a estudiantes que no participan con la finalidad de corroborar la información.

En general los estudiantes participantes de los proyectos se demostraban muy críticos de su formación como docentes, reconocían la falta de una vinculación temprana con los centros educativos como una falencia grave en la formación. Por lo que ven los proyectos de voluntariado como una herramienta útil para lograr una mayor cercanía con la realidad educativa, que les permita conocer sus debilidades y fortalezas, pero además, como una herramienta de trabajo y acción social. 


\subsubsection{Reconocimiento de debilidades y fortalezas}

Al realizar el análisis de los resultados de las encuestas es notable notar que hace un año (antes de iniciar la participación en los proyectos), todos los entrevistados reconocían falencias relacionadas con el manejo de grupo y expresión, ya que eran habilidades que poco se pueden explotar durante la formación académica, pero los resultados cambiaban en gran medida al analizar la visión que tienen de si mismo en relación a su preparación para la docencia, los estudiantes no participante de proyectos sienten que han logrado avances en las áreas que hace un año se sentían débiles, sin incluir nuevas falencias, es decir, plantean una mirada completamente positiva de su formación. Por otra parte los estudiantes participantes de proyectos, sienten que han mejorado sus habilidades, pero que a la vez han reconocido nuevas debilidades, relacionadas directamente con el trabajo en el aula y la organización tanto de contenidos como de tiempos. En general sienten que han mejorado, en gran medida bajo en interés personal de desarrollarse como personas autónomas con el apoyo de los medios que disponen (profesores, textos, compañeros, etc.), pero aun con un largo camino por recorrer, dando el primer paso de reconocer a tiempo sus habilidades reales.

El grupo de entrevistados en general reconocen la necesidad urgente de prácticas tempranas, teniendo en cuentas la precaución de que estas deben ser graduales, viendo en el desarrollo de proyectos una herramienta que tiende a cubrir esta necesidad, ya que en palabras de los participantes esto le permite conocer la realidad de la sala de clases y los problemas que en ella se viven, a pesar de que no sea un contexto completamente real en algunos casos, si da la oportunidad de conocer lo que realmente es la educación, ya no desde el punto de vista de un estudiante, si no de quien se prepara para ser profesor. Así es posible observar las pocas expectativas y la falta de interés en el estudiantado de contextos vulnerables, reconociendo que no se entregan las herramientas para trabajar en estas situaciones.

Cabe señalar que la necesidad de tener espacios donde realizar una especie de practica pasa en primer lugar por la necesidad de conocer la realidad tal como es, no desde la mirada de los autores o la teoría, en segundo lugar, comprobar las capacidades y habilidades personales para desempeñarse como profesor, en tercer lugar, el temor a equivocarse en la elección de futuro, ya que los estudiantes temen esperar llegar a $5^{\circ}$ año y arrepentirse de su elección por no conocer la realidad, y por último, el ser partícipes de una actividad que contribuya a dar mejores oportunidades a quienes no las poseen.

\subsubsection{El rol docente y la formación de una identidad}

Algo de gran importancia en el trabajo de los proyectos es que posiciona al estudiante en un rol distinto, ya desde la mirada del docente que en un futuro será, logrando primero que nada con esto la profundización del rol que cumple el docente, tanto dentro de la sala de clases como en la propia sociedad, en general todos los entrevistados creen en el rol social de docente y su importancia en la formación de la nuevas generaciones, pero quienes han trabajado entienden el rol del docente de forma más compleja y asociado a los contextos de vulnerabilidad, asumiendo que el rol del docente es ayudar en especial a los grupos sociales bajos, buscando para esto las herramientas y conocimientos necesarios. 
Por otra parte, es importante señalar que solo los estudiantes participantes de proyectos tenían una noción de identidad profesional como algo distinto del rol del docente, asumían esto como el estilo personal de trabajo y modos de entender la labor docente en base a las experiencias personales, es decir, con el desarrollo de los proyectos los estudiantes poco a poco van adquiriendo una identidad como docente, la que consideran que es muy básica debido a las poca experiencia, por lo tanto deben seguir formándose, conociendo y desarrollando los conocimientos y experiencias necesarias que les permitan desarrollar su propia identidad.

\section{CONCLUSIONES}

Los resultados de la observación participativa en comunidades educativas diversas y el análisis de las entrevistas a futuros (as) docentes, permiten dar cuenta de una serie de conclusiones que a raíz de este trabajo cobran mayor importancia a la hora de debatir la problemática de la formación inicial docente hoy en día.

Partiendo de la base de que una de las principales críticas que se le hace a la formación inicial docente, que se imparte en la actualidad en muchas universidades y/o centros de educación superior, es que se ha ido transformando en una formación fragmentada en un cúmulo de saberes separados con escasa vinculación entre sí. Particularmente en la formación de docentes en Historia, Geografía y Educación Cívica, se encuentra el caso de que un docente -dependiendo de su afinidad con alguna u otra disciplina- puede saber mucho de historiografía y de procesos históricos, pero no profundiza de la misma manera en los conocimientos de la geografía, pasando por alto muchas veces la multidimensionalidad de los procesos que va enseñar.

Es por ello que el conocer el impacto en la formación profesional docente que tiene el participar en proyectos educativos cobra mayor relevancia, siendo así un real aporte al debate de la problemática de la formación inicial. Siendo esta modalidad de proyectos, un espacio en donde los futuros (as) docentes desarrollan sus competencias disciplinarias y pedagógicas, permitiéndole a ellos (as) descubrir y aplicar simultáneamente a su formación tradicional, gran parte de los elementos que se le enseñan en la teoría; comprendiendo la vinculación de su formación disciplinaria con el contexto donde se va a enseñar, concibiendo esta como un todo interrelacionado. Además de ser también un contexto en donde los futuros profesores, aprenden en la práctica aspectos formales como la gestión tanto organizacional como curricular: desarrollan de buena forma el trabajo en equipo, la organización de comunidades de aprendizaje; así también a través de aprendizaje autónomo y colaborativo se va adquiriendo el conocimiento del currículo, de tal forma que la organización de la clase sea cada vez más planificada y perfectible con los conocimientos formales que se adquieren con la formación profesional.

Otro elemento a considerar, son las competencias transversales que se van adquiriendo con la participación en proyectos educativos. Así se va formando la actitud y el carácter del profesor, factor relevante al momento de enfrentarse a la realidad del mundo laboral. Se va acumulando saberes prácticos que permitirán un mejor desempeño tanto en las prácticas como en el contexto profesional.

Por otro lado, entendiendo el concepto de vinculación temprana, como un primer acercamiento a la realidad de la labor docente, es preciso confirmar la utilidad del 
aprendizaje con servicio como un complemento a la vinculación temprana en la formación inicial docente. Según la opinión recogida, tanto en estudiantes de pedagogía partícipes de proyectos educativos como los que no, estos consideran el aprendizaje con servicio que se concreta en proyectos como los que aquí se mencionan, como una alternativa complementaria, no excluyente, de vinculación temprana en la formación inicial docente.

Se entiende por tanto, que no se está poniendo en duda la formación inicial que entregan las instituciones de educación superior, sino que se plantea la necesidad de mayores instancias de vinculación y/o prácticas tempranas en la misma. Por lo que se valora la existencia de proyectos educativos que entregan la posibilidad a estudiantes que poseen la motivación, la voluntad y el compromiso, además de la inquietud de participar en ellos.

Por último, cabe mencionar los aspectos extrínsecos que trae consigo el aprendizaje con servicio puesto en práctica en los proyectos que sustentan este trabajo. Aspectos como el fortalecimiento de los valores positivos de los estudiantes a quienes se les entrega el servicio; el fomento a la adquisición de saberes y el sentido de pertenencia a las comunidades de las que provienen; se les educa para ser felices; y se les apoya en su desarrollo de la identidad individual y colectiva. Todo lo anterior trasciende a la formación tradicional de los docentes, sino que se relaciona con la formación de profesores cada vez más integrales. Fortaleciendo la vocación docente que todo profesor debiera desarrollar en cuanto a su rol social, como agente de cambio y baluarte de las transformaciones para una sociedad más justa.

\section{REFERENCIAS BIBLIOGRÁFICAS}

Amezcua, M. (2000). El trabajo de campo etnográfico en salud. Una aproximación a la observación participante. Index Enfermería, vol. IX (3), 30-35.

Araujo, A. M. (2004). La pedagogía de la liberación en Paulo Freire. Barcelona: Graò.

Cuevas, F. (2001). Pedagogía anarquista: Textos para la educacion en y para la libertad. Santiago: La Turba Ediciones.

Escamez, J. (1999). Solidaridad y voluntariado social. Valencia: Fundación Bancaja.

Francisco, A. y Moliner, L. (2010). El aprendizaje servicio en la universidad: Una estrategia en la formación de ciudadanía crítica. Revista Electrónica Interuniversitaria de Formación del Profesorado, vol. 13 (4), 69-77.

Freire, P. (2011). Pedagogía del oprimido. México D.F.: Siglo XXI.

García Roca, J. (1994). Solidaridad y voluntariado. Bilbao: Sal Terrae.

Kawulich, B. (2005). La observación participante como método de recolección de datos. Forum: Qualitative Social Research (FQS), vol. 6 (2). Recuperado el 24 de octubre de 2012 desde http:// www.qualitative-research.net/index.php/fqs/article/download/466/999

Labra, P. (2011). Construcción del conocimiento profesional docente: El caso de la formación en la práctica. Tesis para optar al grado de Doctor en Educación. Santiago: Universidad Academia de Humanismo Cristiano.

Martínez, M. (2006). La investigación cualitativa (síntesis conceptual). Revista de Investigción en Psicología (IIPSI), vol. 9 (1), 123-146.

Palomera, R., Fernández-Berrocal, P. y Brackett, M. A. (2008). La inteligencia emocional como competencia básica en la formación inicial de los docentes: Algunas evidencias. Revista Electrónica de Investigación Psicoeducativa, vol. 6 (15), 437-454.

Puig, J. M., Gijón, M., Martín, X. y Rubio, L. (2011). Aprendizaje-servicio y educación para la ciudadanía. Revista de Educación, (Número Extraordinario), 45-67. 
— y Palos, J. (2006). Rasgos pedagógicos del aprendizaje-servicio. Cuadernos de Pedagogía, (357), 60-63.

Tapia, M. N. (2001). La solidaridad como pedagogía. El “aprendizaje-servicio” en la escuela. Buenos Aires: Ciudad Nueva.

Toledo, L. y Reyes, L. (2010). Proyectos de aprendizaje de servicio-comunitario y su influencia en las conductas prosociales de estudiantes universitarios. Revista de Pedagogía, vol. 31 (89), 379-401.

UNESCO. (1998). La educación superior en el siglo XXI. Visión y acción. Recuperado el 24 de octubre de 2012 desde http://www.unesco.org/education/educprog/wche/declaration_spa.htm 